Original Research

\title{
Mindfulness-Based Stress Reduction as a Whole-System Approach to Health Care Delivery: Brief Report from a Pilot Training Program Developed for Clinicians
}

\author{
Holly Hazlett-Stevens *
}

Department of Psychology, University of Nevada, Reno, USA; E-Mail: hhazlett@unr.edu

* Correspondence: Holly Hazlett-Stevens; E-Mail: hhazlett@unr.edu

Academic Editor: Viann N. Nguyen-Feng

Special Issue: Yoga and Mindfulness

OBM Integrative and Complementary Medicine

2021, volume 6, issue 4

doi:10.21926/obm.icm.2104049
Received: July 26, 2021

Accepted: November 14, 2021

Published: November 23, 2021

\begin{abstract}
Mindfulness-Based Stress Reduction (MBSR) is an evidence-based health education program for medical patients with a variety of chronic conditions. MBSR also benefits health care providers themselves, decreasing work-related stress and preventing burnout. The objectives of this study were to describe an abbreviated MBSR curriculum developed for health care providers and to present initial program evaluation data assessing acceptability and outcomes. This program was implemented outside the research context with an open trial design. Mindfulness training sessions were held in a conference room the administrative offices of a large, non-profit community health care system. Five clinic providers, three male and two female, included four primary care physicians and one social worker. All five participants participated in and completed the training. A six-session abbreviated MBSR protocol was developed. Participants attended weekly two-hour group sessions scheduled at the end of the workday. Standardized self-report questionnaires included the Perceived Stress Scale (10-item version) and the Copenhagen Burnout Inventory. Participants also completed four rating items designed to assess their personal satisfaction with the program. All five clinicians reported satisfaction with the program and measurable reductions in perceived stress and personal burnout. Statistically significant reductions in perceived stress and
\end{abstract}

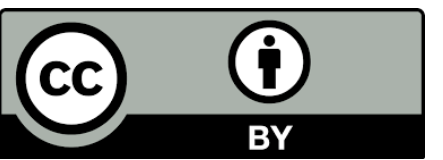


personal burnout scores across the group also were found. Abbreviated MBSR programs for clinicians may support clinician welfare by reducing stress and preventing burnout, ultimately impacting the larger health care delivery system in health-promoting ways. Research examining the effectiveness of specific protocols for clinicians is needed to support further dissemination and implementation.

\section{Keywords}

Mindfulness; MBSR; stress; burnout

\section{Introduction}

Jon Kabat-Zinn challenged the traditional health care paradigm almost 40 years ago with the development of his Stress Reduction Clinic at the University of Massachusetts (UMass) Medical School. In 1979, Kabat-Zinn first implemented his eight-week intensive mindfulness meditation and yoga course within this mainstream American general hospital setting, and dozens of randomized controlled trials since have demonstrated the effectiveness of this Mindfulness-Based Stress Reduction (MBSR) curriculum for a variety of medical and psychiatric conditions [1].

Kabat-Zinn's pioneering work was guided by a significantly different paradigm, characterized by increased patient participation and a central role for the doctor-patient relationship. This participatory medicine model contrasted sharply with views of the patient as a passive recipient of health care driven by an authoritative physician. From this newer perspective, medicine allows for profound healing of the whole person and is not limited to quick fixes or cures. Thus, MBSR represented a radical departure from traditional models in which medicine merely "fixed" specific symptoms or subsystems in isolation without viewing the person as a whole. Indeed, Kabat-Zinn [2] originally cited the Self-Regulation Theory of Gary Schwartz [3], viewing health as comprised of interconnected complex whole systems that cannot be reduced into component parts to be treated in isolation. MBSR therefore taught patients to see themselves as whole person systems who are part of a larger health care system in which they play an active role to optimize their own health and healing. Mindfulness, meditation, and mind-body medicine may be imperative to health care reform efforts that aim to reduce the demand for costly services by leveraging preventive medicine [4].

In another important development, physicians and other health care staff eventually attended MBSR courses themselves for their own stress reduction, health, and well-being [5]. As they observed their patients assume greater responsibility for their own health and approach the doctorpatient relationship more as participants than as passive recipients, physicians and nurses began to follow suit. This trend not only shifted physicians' understanding of their own humanity, but also encouraged views of the healing relationship as a collaboration between patient and provider [6]. Increased mindfulness among providers may reduce the likelihood of patients coming away from an encounter feeling disregarded or misunderstood, and mindful medical practice can improve the quality of care directly as providers engage in critical self-reflection to recognize their own errors and to clarify decision-making processes [7]. In this way, MBSR ultimately impacted larger interpersonal and social systems within the health care field. From this perspective, value-based 
medical delivery can be viewed as the larger whole within which individual patient and provider whole-person systems are embedded. Not surprisingly, mindfulness training has become a popular and effective component of medical education, appearing in medical school curricula [8] and routinely offered in continuing medical education training programs.

A growing body of research has demonstrated benefits of the eight-week MBSR curriculum among health care providers [9-12]. Mindfulness training generally improved the psychological functioning of health care staff, increased empathy, and improved the quality of interpersonal relations and interactions with others [13]. However, implementation of the full eight-week MBSR curriculum within the medical work setting may not be practicable for many busy health care professionals. Fortunately, abbreviated protocols also may be effective for clinicians, such as one involving only four weekly 90-minute mindfulness training sessions and one six-hour all-day retreat [14]. Another effective program delivered mindfulness training to clinicians over 18 hours of total session time scheduled during an intensive weekend period ( 3 hours Friday evening, 7 hours Saturday, 4 hours Sunday) plus two subsequent two-hour evening sessions within five weeks after the initial weekend sessions [15]. While these abbreviated curricula provide initial guidance to medical center administrators looking to implement a more practical form of MBSR for their staff, each requires medical staff to devote an entire day or weekend of personal time to mindfulness training. A third promising abbreviated MBSR curriculum developed for health care employees in Columbia consisted of only four weekly two-hour sessions [16], but this protocol has not been widely studied. Health care delivery systems that aim to promote mindfulness within the entire system may require MBSR protocol flexibility regarding the total amount of session time, length of sessions, and/or scheduling times in order to meet the needs of participating health care providers.

The current report describes an alternative abbreviated MBSR curriculum, developed to meet the needs of medical providers within a large non-profit medical organization that offer a range of health care services to underserved communities. This twelve-hour abbreviated MBSR program was delivered over six weekly two-hour sessions to a small group of clinicians immediately after their workday. Preliminary program evaluation data assessing providers' satisfaction with the program and measuring changes in provider perceived stress and burnout were collected. Results from this initial program evaluation are reported following an Institutional Review Board human subjects determination review that allowed for the publication of these data.

\section{Method}

\subsection{Participants and Procedure}

A free abbreviated MBSR course was offered to clinic health care providers at a large non-profit community health care system. Five experienced clinicians (three male and two female), comprised of four primary care physicians and one social worker, volunteered to attend the training. Based upon informal input collected from these participants, the MBSR program was delivered over six weekly two-hour sessions, scheduled immediately after the end of the workday from 6 to $8 \mathrm{pm}$. Each session took place at the same time on the same day of the week, once per week, over six consecutive weeks. These clinicians opted to attend the training based upon a written description of the program. For this reason, the first session provided introductory orientation information about MBSR before presentation of the standard curriculum components typically presented in the first MBSR session. All MBSR sessions were facilitated by the first author, a licensed psychologist 
with over twelve years of personal meditation practice who had completed all formal training programs in MBSR provided by the Oasis Institute for Mindfulness-Based Professional Education at the UMass Center for Mindfulness in Medicine, Health Care, and Society. The MBSR instructor adapted the standard curriculum outlined in the UMass Center for Mindfulness 2009 Curriculum Guide [17] into an abbreviated six-session protocol. Table 1 provides an outline of specific MBSR curriculum components included in each session.

Table 1 Specific MBSR curriculum components delivered in each weekly session.

\begin{tabular}{|c|c|c|}
\hline Session number & Curriculum activity & Duration of activity \\
\hline \multirow[t]{5}{*}{ Session 1} & $\begin{array}{l}\text { Pre measures collected, introduction to MBSR, and } \\
\text { "raisin exercise" }\end{array}$ & $30 \mathrm{~min}$ \\
\hline & Awareness of breath meditation & $10 \min$ \\
\hline & Group member introductions & $35 \mathrm{~min}$ \\
\hline & Body scan formal meditation & $40 \mathrm{~min}$ \\
\hline & Presentation of home practice assignments & $5 \mathrm{~min}$ \\
\hline \multirow[t]{4}{*}{ Session 2} & Mindful movement and body scan practice & $45 \min$ \\
\hline & Group discussion of practice; introduce "STOP" & $45 \min$ \\
\hline & $\begin{array}{l}\text { Didactic presentation of "9-dots exercise" and } \\
\text { "Upstream/Downstream fable" }\end{array}$ & $20 \mathrm{~min}$ \\
\hline & $\begin{array}{l}\text { Closing breath meditation and present home practice } \\
\text { assignments }\end{array}$ & $10 \mathrm{~min}$ \\
\hline \multirow[t]{4}{*}{ Session 3} & $\begin{array}{l}\text { Mindful movement and sitting meditation including } \\
\text { breath, body sensations, and sound }\end{array}$ & $45 \mathrm{~min}$ \\
\hline & Group discussion of practice; introduce "RAIN" & $45 \mathrm{~min}$ \\
\hline & Review pleasant events calendar & $20 \mathrm{~min}$ \\
\hline & Brief breath meditation and present home practice & $10 \mathrm{~min}$ \\
\hline \multirow[t]{6}{*}{ Session 4} & Full sitting meditation sequence & $35 \mathrm{~min}$ \\
\hline & Participant-guided mindful movement & $10 \mathrm{~min}$ \\
\hline & Walking meditation instructions and practice & $15 \min$ \\
\hline & Group discussion of practice & $20 \mathrm{~min}$ \\
\hline & $\begin{array}{l}\text { Review unpleasant events calendar and discuss the } \\
\text { nature of stress }\end{array}$ & $30 \mathrm{~min}$ \\
\hline & Brief breath meditation and present home practice & $10 \mathrm{~min}$ \\
\hline \multirow[t]{5}{*}{ Session 5} & Full sitting meditation sequence & $30 \mathrm{~min}$ \\
\hline & Walking meditation & $10 \mathrm{~min}$ \\
\hline & Lovingkindness meditation & $20 \mathrm{~min}$ \\
\hline & Group discussion of practice & $20 \mathrm{~min}$ \\
\hline & $\begin{array}{l}\text { Continued discussion of stress didactic material, } \\
\text { review difficult communications calendar, engage } \\
\text { class in communication styles activity }\end{array}$ & $40 \mathrm{~min}$ \\
\hline \multirow[t]{2}{*}{ Session 6} & Body scan, mindful movement, sitting meditation & $60 \mathrm{~min}$ \\
\hline & Group discussion of practice & $20 \min$ \\
\hline
\end{tabular}


Complete post measures and course evaluation $40 \mathrm{~min}$

questions, followed by final discussion

\subsection{Measures}

All five clinicians completed standardized self-report measures of perceived stress (10-item Perceived Stress Scale-PSS [18]) and clinician burnout (Copenhagen Burnout Inventory-CBI [19]) at the beginning of the first MBSR session and during the final sixth MBSR session. The PSS contains items such as "In the last week, how often have you been upset because of something that happened unexpectedly?" and "In the last week, how often have you been angered because of things that were outside your control?" Participants answer each item on a Likert scale of 0 to 4, where $0=$ never, 1 = almost never, 2 = sometimes, 3 = fairly often, and 4 = very often. Reversedscored item values are reversed, and then item scores are summed to create a total score. The CBI is a nineteen-item measure with subscales assessing: 1) personal burnout, 2) work-related burnout, and 3) patient-related burnout, defined as a state of prolonged physical and psychological exhaustion perceived as related to one's work with patients. CBI items include "How often are you emotionally exhausted?" (personal burnout), "Do you feel worn out at the end of the working day?" (work-related burnout), and "Do you find it frustrating to work with patients?" (patient-related burnout). Response options include "always," "often," sometimes," "seldom," and "never/almost never." Scores for each scale are obtained by averaging responses across items on that scale, in which $100 \%$ corresponds to "always," $75 \%$ corresponds to "often," $50 \%$ corresponds to "sometimes," 25\% corresponds to "seldom," and 0\% corresponds to "never/almost never." Thus, possible scores for each scale range from 0-100. Both the PSS and the CBI are widely used measures with substantial research evidence demonstrating good reliability and validity $[18,19]$.

During the final MBSR session, clinicians also gave response ratings to four survey questions written by the author for this pilot investigation: 1) "How helpful was this stress reduction class for you?" 2) "Has this stress reduction class reduced your overall level of stress?" 3) "Have you noticed improvement in the ways you handle stressful events in your life?" 4) "Has this stress reduction class improved your overall quality of life and/or enjoyment of life?" Responses used a 1-10 Likert scale in which $1=$ "Not at all" and $10=$ "Extremely."

\section{Results}

\subsection{Perceived Stress}

Paired-samples t-test conducted with PSS-10 scores collected during the first MBSR session ( $M=$ 21.60; $S D=6.11)$ and repeated during the last MBSR session $(M=14.20 ; S D=3.03)$ revealed that this observed decrease was statistically significant $[t(4)=4.10, p<.015$ (two-tailed); Cohen's $d=$ 1.53]. Inspection of individual scores revealed that four of the five clinicians scored more than one standard deviation above published normative sample means [18] before MBSR, yet all four scored near the mean and within one standard deviation post-MBSR. The fifth clinician scored at the normative sample mean pre-MBSR but scored below one-half of the standard deviation below that mean post-MBSR. Thus, each individual clinician reported meaningful reductions in perceived stress after MBSR, and all five scored within the normal range on this measure at the final session. 


\subsection{Clinician Burnout}

The observed reduction in $\mathrm{CBI}$ personal burnout subscale scores pre-MBSR $(M=57.00 ; S D=$ $17.89)$ to post-MBSR $(M=38.00 ; S D=9.08)$ also reached statistical significance $[t(4)=2.97, p<.041$ (two-tailed); Cohen's $d=1.34$ ]. Importantly, the pre-MBSR mean on this subscale was elevated well above one standard deviation above normative sample means [19], but the mean on this scale fell well within normal limits post-MBSR. The smaller reductions in CBI work burnout subscale scores pre-MBSR $(M=57.80 ; S D=16.18)$ to post-MBSR $(M=45.60 ; S D=10.48)$ and in CBI patient burnout subscale scores pre-MBSR $(M=42.60 ; S D=14.50)$ to post-MBSR $(M=33.40 ; S D=17.04)$ failed to reach statistical significance $\left(p^{\prime} s<.143\right.$ and .086 , respectively; Cohen's $d=0.90$ and 0.58 , respectively). However, comparison of these means to published normative data reveal that the reduction observed on the work burnout subscale also crossed over a threshold from an elevated mean score pre-MBSR to a mean score within one standard deviation of the normative sample mean post-MBSR. The patient burnout subscale mean score was within the normative range both before and after MBSR. Each CBI subscale score reflects an average rating of items from that subscale, such that a frequency of "sometimes" is captured by a score of 50 . The observation that each $\mathrm{CBI}$ subscale mean score fell below 50 post-MBSR therefore reflected that clinicians reported they experienced various symptoms of burnout less often than "sometimes" on average. For this reason, scores below 50 on the $\mathrm{CBI}$ subscales are considered to reflect an absence of clinician burnout [19]. In addition, inspection of individual scores revealed that all clinicians reported reductions in $\mathrm{CBI}$ scores pre to post-MBSR, suggesting that each experienced some degree of reduced burnout. See Table 2 for individual scores pre and post for each outcome measure.

Table 2 Individual participant scores immediately before and at the end of the MBSR program.

\begin{tabular}{lllllllll}
\hline Participant & PSS & PSS & CBIP & CBIP & CBIW & CBIW & CBIC & CBIC \\
& Pre & Post & Pre & Post & Pre & Post & Pre & Post \\
\hline$\# 1$ & 26 & 17 & 70 & 50 & 68 & 64 & 50 & 54 \\
$\# 2$ & 28 & 15 & 75 & 35 & 79 & 43 & 42 & 21 \\
$\# 3$ & 18 & 15 & 60 & 45 & 46 & 43 & 63 & 50 \\
$\# 4$ & 23 & 15 & 50 & 30 & 57 & 39 & 29 & 21 \\
$\# 5$ & 13 & 9 & 30 & 30 & 39 & 39 & 29 & 21 \\
\hline
\end{tabular}

Note. PSS $=10$-item Perceived Stress Scale score; CBIP = Copenhagen Burnout InventoryPersonal burnout score;

$\mathrm{CBIW}=$ Copenhagen Burnout Inventory-Work burnout score; $\mathrm{CBIC}=$ Copenhagen Burnout Inventory-Patient burnout score

\subsection{Satisfaction Ratings}

When asked "How helpful was this stress reduction class for you?" clinicians responded with a rating that ranged from 7 to 10 on a ten-point Likert scale $(M=8.40 ; S D=1.34)$. In response to the question "Has this stress reduction class reduced your overall level of stress?" clinician ratings ranged from 6 to $10(M=7.80 ; S D=1.79)$. The question "Have you noticed improvement in the ways 
you handle stressful events in your life?" yielded ratings from 5 to $10(M=7.80 ; S D=1.79)$, and the final question, "Has this stress reduction class improved your overall quality of life and/or enjoyment of life?" resulted in ratings ranging from 6 to $10(M=8.40 ; S D=1.67)$.

\section{Discussion}

This initial implementation of an abbreviated MBSR curriculum involved a small group of clinicians who provide care to patients within a large, non-profit community health care clinic. Participating clinicians reported that each experienced reductions in perceived stress and burnout and noticed signs of improvement in their lives at the end of the program. Observed reductions on measures of perceived stress and personal burnout across participants were statistically significant, and effect size statistics revealed a large effect for both measures. These reductions appear meaningful beyond statistical significance, as the mean scores on all measures fell within normative ranges and suggested an absence of burnout at the conclusion of the six-week MBSR program. All five clinicians completed the program, and all clinicians anecdotally reported that they had a positive experience with the program without any adverse effects. Thus, initial program evaluation results from this pilot program suggest that this abbreviated protocol format may provide an effective alternative to the full MBSR curriculum for clinicians when delivered within health care delivery systems.

Nevertheless, these results are preliminary and several limitations should be considered. First, the durability of the immediate improvements found in this study is unknown, as follow-up data were not collected. Second, the extremely small sample studied in this initial pilot investigation leaves the statistically significant findings difficult to interpret. Furthermore, these five clinicians all volunteered to attend the MBSR program as additional training after their workday had ended. Therefore, it is unclear how much these results generalize to other health care providers who do not self-select into MBSR and who participate in MBSR as required staff trainings conducted during the workday instead. Controlled research examining the effectiveness of this particular MBSR protocol among larger samples of providers is needed. Comparison with a randomized notreatment or waitlist control condition would suggest that any benefits following this MBSR protocol were due to the program itself rather than to other extraneous factors.

Future research examining the mechanisms through which MBSR decreases perceived stress and burnout among clinicians might be beneficial, leading to the identification of which components or aspects of the program are most responsible for observed positive effects. Another future research direction involves study of other available abbreviated MBSR protocols, as a variety of alternative MBSR protocols may be necessary for broader scale implementation of MBSR for clinicians both within and across health care delivery systems. Given the wide variation in clinician schedules, personal and family obligations outside the workplace, and levels of interest and/or motivation to attend mindfulness training, the availability of multiple protocol options potentially could maximize dissemination of mindfulness training for clinicians. Making MBSR available and easily accessible not only to patients, but to medical staff as well, would allow for a whole systems research approach that evaluates the impact of mindfulness across levels within value-based medical delivery systems. 


\section{Acknowledgement}

This work was conducted at the Community Health Alliance in Reno, NV. The author gratefully acknowledges Dr. Dan Ahearn, Chuck Duarte, and all Community Health Alliance administrative staff who made this project possible. No grants or other financial support was used.

\section{Author Contributions}

The author did all the research work of this study.

\section{Competing Interests}

The author has declared that no competing interests exist.

\section{References}

1. de Vibe M, Bjørndal A, Fattah S, Dyrdal GM, Halland E, Tanner-Smith EE. Mindfulness-based stress reduction (MBSR) for improving health, quality of life and social functioning in adults: $A$ systematic review and meta-analysis. Campbell Syst Rev. 2017; 13: 1-264.

2. Kabat-Zinn J. Full catastrophe living: Using the wisdom of your body and mind to face stress, pain, and illness. New York: Bantam Books; 1990.

3. Schwatrz GE. Psychobiology of health: A new synthesis. In: Psychology and health: Master lecture series. Wahsington: American Psychological Association; 1984.

4. Ruff KM, Mackenzie ER. The role of mindfulness in healthcare reform: A policy paper. Explore. 2009; 5: 313-323.

5. Kabat-Zinn J. Coming to our senses: Healing ourselves and the world through mindfulness. New York: Hyperion; 2005.

6. Santorelli S. Heal thy self: Lessons on mindfulness in medicine. New York: Three Rivers Press; 1999.

7. Epstein RM. Mindful practice. JAMA. 1999; 282: 833-839.

8. Hassed C, De Lisle S, Sullivan G, Pier C. Enhancing the health of medical students: Outcomes of an integrated mindfulness and lifestyle program. Adv Health Sci Educ. 2009; 14: 387-398.

9. Shapiro SL, Schwartz GE, Bonner G. Effects of mindfulness-based stress reduction on medical and premedical students. J Behav Med. 1998; 21: 581-599.

10. Martín-Asuero A, García-Banda G. The mindfulness-based stress reduction program (MBSR) reduces stress-related psychological distress in healthcare professionals. Span J Psychol. 2010; 13: 897-905.

11. Galantino ML, Baime M, Maguire M, Szapary PO, Farrar JT. Association of psychological and physiological measures of stress in health-care professionals during an 8-week mindfulness meditation program: Mindfulness in practice. Stress Health. 2005; 21: 255-261.

12. Shapiro SL, Astin JA, Bishop SR, Cordova M. Mindfulness-based stress reduction for health care professionals: Results from a randomized trial. Int J Stress Manag. 2005; 12: 164-176.

13. Escuriex BF, Labbé EE. Health care providers' mindfulness and treatment outcomes: A critical review of the research literature. Mindfulness. 2011; 2: 242-253. 
14. Jain S, Shapiro SL, Swanick S, Roesch SC, Mills PJ, Bell I, et al. A randomized controlled trial of mindfulness meditation versus relaxation training: Effects on distress, positive states of mind, rumination, and distraction. Ann Behav Med. 2007; 33: 11-21.

15. Fortney L, Luchterhand C, Zakletskaia L, Zgierska A, Rakel D. Abbreviated mindfulness intervention for job satisfaction, quality of life, and compassion in primary care clinicians: $A$ pilot study. Ann Fam Med. 2013; 11: 412-420.

16. Manotas M, Segura C, Eraso M, Oggins J, McGovern K. Association of brief mindfulness training with reductions in perceived stress and distress in Colombian health care professionals. Int J Stress Manag. 2014; 21: 207-225.

17. Santorelli SF, Kabat-Zinn J. Mindfulness-based stress reduction (MBSR) professional education and training: MBSR curriculum and supporting materials. Boston: Center for Mindfulness; 2009.

18. Cohen S, Williamson G. Perceived stress in a probability sample of the U.S. In: The social psychology of health: Claremont symposium on applied social psychology. Newbury Park: Sage; 1988.

19. Kristensen TS, Borritz M, Villadsen E, Christensen KB. The Copenhagen burnout inventory: A new tool for the assessment of burnout. Work Stress. 2005; 19: 192-207.

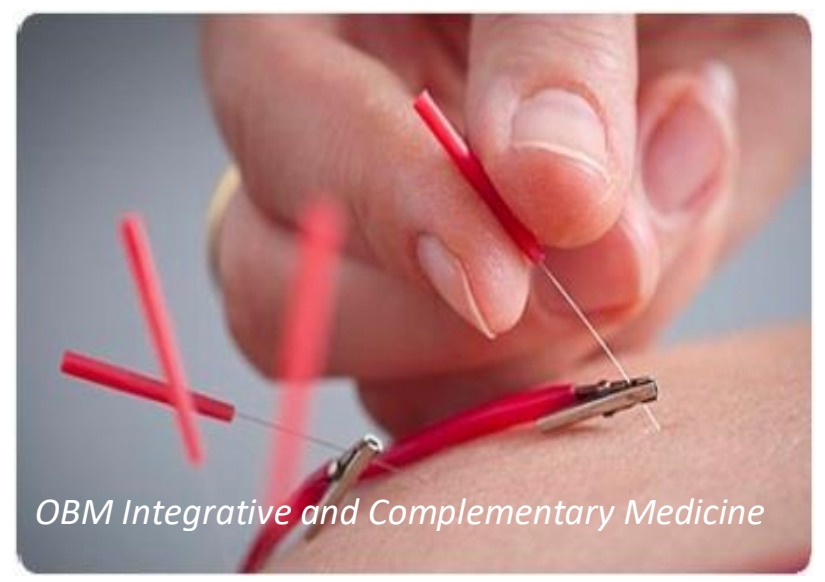

Enjoy OBM Integrative and Complementary Medicine by:

1. Submitting a manuscript

2. Joining in volunteer reviewer bank

3. Joining Editorial Board

4. Guest editing a special issue

For more details, please visit: http://www.lidsen.com/journals/icm 